UCRL-JC-129119

PREPRINT

\title{
Analysis of Intergranular Impurity Concentration and the Effects on the Ductility of Copper Shaped Charge Jets
}

\author{
Adam J. Schwartz \\ David H. Lassila \\ Ernest L. Baker
}

This paper was prepared for submittal to Ballistics '98 17th International Symposium on Ballistics Midrand, South Africa

March 23-27, 1998

November 20, 1997

This is a preprint of a paper intended for publication in a joumal or proceedings. Since changes may be made before publication, this preprint is made available with the understanding that it will not be cited or reproduced without the permission of the author. 


\section{DISCLAIMER}

This document was prepared as an account of work sponsored by an agency of the United States Government. Neither the United States Government nor the University of California nor any of their employees, makes any warranty, express or implied, or assumes any legal liability or responsibility for the accuracy, completeness, or usefulness of any information, apparatus, product, or process disclosed, or represents that its use would not infringe privately owned rights. Reference herein to any specific commercial product, process, or service by trade name, trademark, manufacturer, or otherwise, does not necessarily constitute or imply its endorsement, recommendation, or favoring by the United States Government or the University of California. The views and opinions of authors expressed herein do not necessarily state or reflect those of the United States Government or the University of California, and shall not be used for advertising or product endorsement purposes. 


\title{
ANALYSIS OF INTERGRANULAR IMPURITY CONCENTRATION AND THE EFFECTS ON THE DUCTILITY OF COPPER SHAPED CHARGE JETS
}

\author{
Adam J. Schwartz (1), David H. Lassila (1), and Ernest L. Baker (2) \\ (1) Lawrence Livermore National Laboratory, L-355, P.O. Box, 808, Livermore, CA 94550 \\ (2) U.S. Army, TACOM-ARDEC, Picatinny, NJ 07806
}

\begin{abstract}
A geometrical analysis based on an assumed tetrakaidecahedron grain shape is applied to determine the relationship among grain size, bulk impurity content, and breakup time in sulfur-doped, high-precision, 81-mm, oxygen-free electronic (ofe) copper shaped charge liners. The calculations determine the number of impurity atoms as a function of grain size, the number of available sites at the intercrystalline defects, and the intercrystalline impurity concentration. Recent experiments have shown that some larger grain size liners with low impurity contents exhibit better ductility than smaller grain size liners with higher impurity concentrations. Within the range of grain sizes and bulk impurity contents in this study, the analysis suggests that the quadruple nodes and triple lines are saturated with impurities. Over this same range of impurities and grain sizes, only partial filling of a monolayer of impurities exists at the grain boundaries. The analysis suggests that breakup time is fundamentally related to grain boundary impurity concentration.
\end{abstract}

\section{INTRODUCTION}

There has been significant discussion in the shaped charge literature regarding the importance of grain size and impurity content on the ductility of shaped charge jets. In the majority of these investigations, one of these parameters was held constant, while the other was varied. Duffy and Golaski found that at constant impurity content, jet ductility increases as the grain size decreases [1]. An additional observation has been that at constant grain size, jet ductility improves as the bulk impurity content decreases [2,3]. Lichtenberger has proposed a criterion for the selection of shaped charge liner materials based on the recrystallization temperature $[4,5]$, which indirectly takes into account the relationship between grain size and impurity content. Despite these general trends in the experimental observations of jet ductility, there have been no systematic studies aimed at determining the combined effects of grain size and impurity content on breakup behavior. The purpose of this investigation is to examine the relationship among grain size, overall impurity content, and shaped charge jet ductility in sulfur-doped oxygen free electronic (ofe) copper. The analysis was motivated by experiments which indicate that large grain size liners sometimes exhibit better ductility than small grain size liners. Similarly, longer breakup times have been observed in some lower purity materials than in higher purity materials (see Fig. 3).

It is well known that impurities such as oxygen [6], sulfur [7], and bismuth [8] cause embrittlement in copper. In order to evaluate the influence of impurity content on the behavior of shaped charge jets, a knowledge of the spatial distribution of impurities is necessary. In a two-dimensional representation of the microstructure, i.e., a micrograph, grain boundaries are observed as lines, and triple lines as points. In an actual material, grain boundaries are surfaces separating two misoriented grains, triple lines are linear defects occurring at the intersection of three grains, and quadruple nodes are the point intersection of four triple lines. Depending on a number of factors, however, each of these microstructural features can be assumed to occupy some volume. For example, the grain boundary can be assumed to have some width, and triple lines a length times a cross sectional area. The intersection of four triple lines at a quadruple node would then lead to a volume for the quadruple node, as discussed by Palumbo et al. [9] and Wang et al. [10]. The critical assumption of this analysis is that all impurity atoms diffuse along, and remain at, the grain boundaries, triple lines, and/or quadruple nodes. 


\section{EXPERIMENTAL PROCEDURES}

The copper shaped-charge liners were produced from ofe 99.99\% copper, Hitachi C10100 bar stock with the measured impurity concentration obtained by chemical analysis listed in Table 1 [11]. The liners were back extruded using a standard cold-forge process into the shape of hollow cones (base inner diameter $=81 \mathrm{~mm}$, apex angle $=42^{\circ}$ ). After forging, the liners were annealed at $315^{\circ} \mathrm{C}$ for one hour or $400^{\circ} \mathrm{C}$ for 10 minutes or 100 hours in order to stabilize the microstructure for subsequent sulfur doping. After the recrystallization anneal, several of the liner cones were doped with sulfur as described in Refs. [2,3]. The grain size of the liners was measured using standard metallographic techniques, and the breakup times determined from the flash $\mathrm{x}$-ray radiographs of the jets. Shot data for high impurity content liners $(10-15 \mathrm{ppm} \mathrm{S})$ is reported for the first time.

Table 1. Measured impurity concentrations in ofe copper, Hitachi C10100 stock. All other elements had concentrations $<0.1 \mathrm{ppm}$.

\begin{tabular}{|c|c|c|c|}
\hline Impurity & $\begin{array}{c}\text { Concentration } \\
(\mathrm{ppm})\end{array}$ & Impurity & $\begin{array}{c}\text { Concentration } \\
(\mathrm{ppm})\end{array}$ \\
\hline $\mathrm{H}$ & 0.9 & $\mathrm{Ni}$ & 1.0 \\
\hline $\mathrm{C}$ & 5.0 & $\mathrm{As}$ & 0.4 \\
\hline $\mathrm{N}$ & $<0.1$ & $\mathrm{Se}$ & 0.3 \\
\hline $\mathrm{O}$ & 6.0 & $\mathrm{Ag}$ & 6.4 \\
\hline $\mathrm{Si}$ & 0.2 & $\mathrm{Sb}$ & 0.3 \\
\hline $\mathrm{P}$ & 0.4 & $\mathrm{~Pb}$ & 0.2 \\
\hline $\mathrm{S}$ & 4.0 & $\mathrm{Bi}$ & 0.2 \\
\hline $\mathrm{Fe}$ & 2.0 & - & - \\
\hline
\end{tabular}

\section{SIMULATION OF THE MICROSTRUCTURE}

To simulate the microstructure for analysis, a number of approaches may be taken. The basic methods would be to approximate the grains as cubes or spheres. Cubic grains represent the simplest of approaches, but real materials rarely have grain morphologies which can be approximated as cubes. An advantage of using spheres to approximate the grain morphologies is that a grain size distribution can be incorporated. In this case, the distribution of sphere sizes can be made to touch, thus leaving a small amount of free volume, or made to overlap, leading to an overestimate of the volume giving rise to upper and lower bounds of surface area to volume ratios. A disadvantage of the spherical approach is the difficulty in calculating grain boundary area and "triple line" lengths.

The approach used in this analysis is to approximate the shape of the grains by truncated octahedra, or Kelvin tetrakaidecahedra as shown in Fig. 1. A tetrakaidecahedron is a 14-sided polyhedra of which the faces consist of 6 squares and 8 hexagons formed by truncating two opposing pyramids. Each face (hexagon and square) has one full-facing neighbor of the same configuration in the adjacent grain, while each line of length $\lambda$ is the boundary between three tetrakaidecahedron grains. The advantages of using tetrakaidecahedra are that the objects fill space entirely and come close to minimizing the surface area to volume ratio. A disadvantage of this approach is that a grain size distribution cannot be considered.

It is necessary to relate the characteristic length of the tetrakaidecahedron, $\lambda$, to the diameter of the grains, $d$, in the material. A lower value would be to use the diameter of an inscribed sphere; however, for this analysis, an upper limit is applied. The diameter of the tetrakaidecahedron is calculated at the maximum spanning length in the cell. From vertex to vertex:

$$
d=\sqrt{10} \lambda
$$




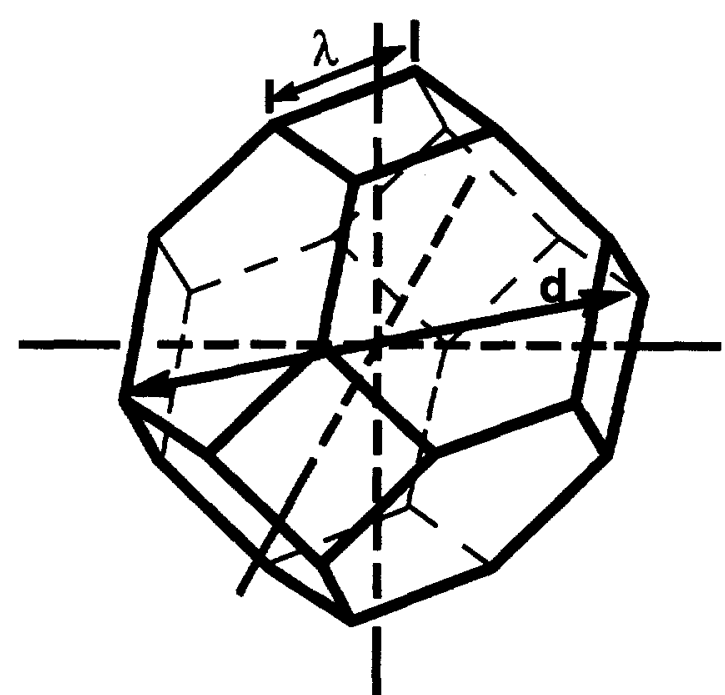

(a)

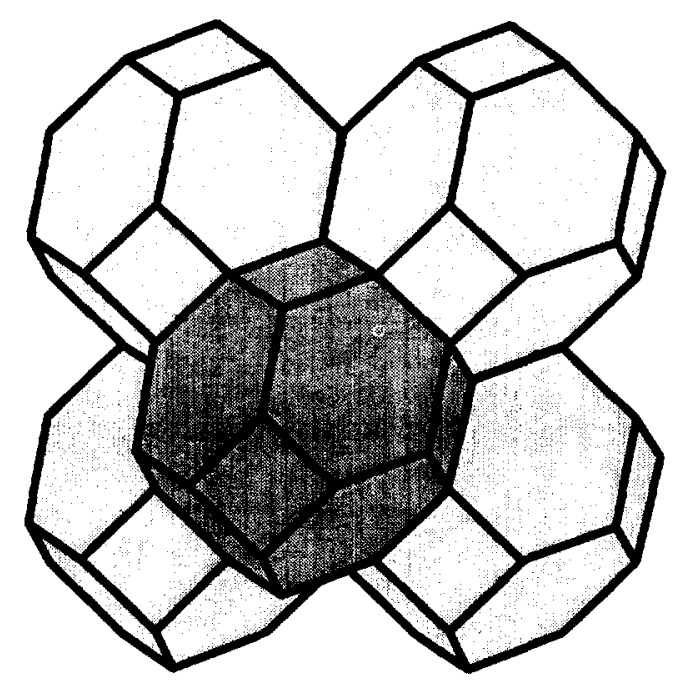

(b)

Figure 1. (a) Isolated tetrakaidecahedron. This 14-sided polyhedra has a characteristic length, $\lambda$, and consists of 6 square and 8 hexagonal faces. (b) Assembly of tetrakaidecahedra. Each individual tetrakaidecahedron has 14 neighbors, each sharing one face with the opposing grain.

When the individual tetrakaidecahedra are assembled to entirely fill space in a "body-centered cubic like" packing arrangement as shown in Fig. 1(b), the equations for volume, surface area, triple line length, and number of quadruple nodes must be modified to allow for the "sharing" of microstructural features between the grains. The volume of the grain remains unchanged, but since each face of the tetrakaidecahedron is shared with another, the surface area per grain in the assembly is reduced by a factor of two. Similarly, since each triple line represents the linear boundary between three grains, the length of triple lines per grain in the assembly is reduced by a factor of three. Each quadruple node is shared by four grains, thus the individual tetrakaidecahedron submits six quadruple nodes per grain to the entire assembly. The volume, surface area, and length of triple lines for an assembly of tetrakaidecahedra in terms of the characteristic length, $\lambda$, and the grain size, $d$, is as follows:

$$
\begin{aligned}
& V=8 \sqrt{2} \lambda^{3}=\frac{4 \sqrt{5}}{25} d^{3} \\
& A=(3+6 \sqrt{3}) \lambda^{2}=\frac{(3+6 \sqrt{3}) d^{2}}{10} \\
& L=12 \lambda=\frac{12 d}{\sqrt{10}}
\end{aligned}
$$

\section{INTERGRANULAR IMPURITY CONCENTRATION}

The method used to determine the "effective" concentration of impurities at the grain boundaries, triple lines, and quadruple nodes begins by calculating the total number of available atomic sites at these intercrystalline defects. The impurity concentration at each location then equals the total number of impurities (in each grain), $\mathbf{N}_{i}$, divided by the total number of available sites as a function of grain size. Taking the lattice parameter of fcc copper as: $a_{0}=$ $0.362 \mathrm{~nm}$, the volume per atom, $\mathrm{V}_{\mathrm{a}}$ is calculated as:

$$
\mathrm{V}_{\mathrm{a}}=\mathrm{a}_{\mathrm{o}}^{3} / 4
$$

The total number of atoms in this volume, $\mathrm{N}_{\mathrm{a}}$, is equal to volume of the grain (grain size, $\mathrm{d}$ ) divided by the volume per atom: 


$$
\mathrm{N}_{\mathrm{a}}=\frac{\mathrm{V}_{\mathrm{g}}}{\mathrm{V}_{\mathrm{a}}}=\frac{16 \mathrm{~d}^{3}}{5 \sqrt{5} \mathrm{a}_{\mathrm{o}}^{3}},
$$

and the total number of impurity atoms equals the concentration of impurities, $\mathrm{C}_{\mathrm{s}}$, times the total number of atoms,

$$
\mathrm{N}_{\mathrm{i}}=\mathrm{C}_{\mathrm{s}} \mathrm{N}_{\mathrm{a}}=\frac{\mathrm{C}_{\mathrm{s}} \mathrm{V}_{\mathrm{g}}}{\mathrm{V}_{\mathrm{a}}}=\frac{16 \mathrm{C}_{\mathrm{s}} \mathrm{d}^{3}}{5 \sqrt{5} \mathrm{a}_{\mathrm{o}}^{3}} \text {. }
$$

Grain Boundary Impurity Concentration: The concentration of impurities at the grain boundaries is determined by distributing a monolayer of impurities over the entire grain boundary area: assuming the impurity atoms are arranged in a hexagonal packing arrangement. The calculation for the area per atom uses the atomic radius of sulfur, $r_{s}=0.127 \mathrm{~nm}$.

$$
\mathrm{A}_{\text {atom }}=2 \sqrt{3} \mathrm{r}_{\mathrm{s}}^{2}
$$

Thus, the total number of sites at the grain boundary is given by:

$$
\text { Sites }_{g b}=\frac{A_{g b}}{A_{\text {atom }}}=\frac{(3+6 \sqrt{3}) d^{2}}{20 \sqrt{3} r_{s}^{2}} \text {. }
$$

The possible concentration of impurities at the grain boundary is:

$$
C_{g b}=\frac{N_{i}}{\text { Sites }_{g b}}=\frac{64 \sqrt{6} r_{s}^{2} C_{s} d}{(3+6 \sqrt{3}) \sqrt{10 a_{o}^{3}}} .
$$

Figure 2 represents the concentration of impurities at the grain boundaries (as a fraction of one monolayer coverage) as a function of grain size, for differing levels of bulk impurity content as derived in Eq. (10). To calculate the grain size at which the concentration of impurities at the grain boundaries saturates at $1 \mathrm{ppm}$ bulk impurity content, set $\mathrm{C}_{\mathrm{gb}}=1.0$ and $\mathrm{C}_{\mathrm{s}}=1 \mathrm{ppm}$ $\left(1 \times 10^{-6}\right)$. At $1 \mathrm{ppm}$ bulk impurity content, the grain boundaries will saturate with impurities for d $>794.54 \mu \mathrm{m}$. At $10 \mathrm{ppm}$, the grain boundaries saturate at $\mathrm{d}>79.45 \mu \mathrm{m}$.

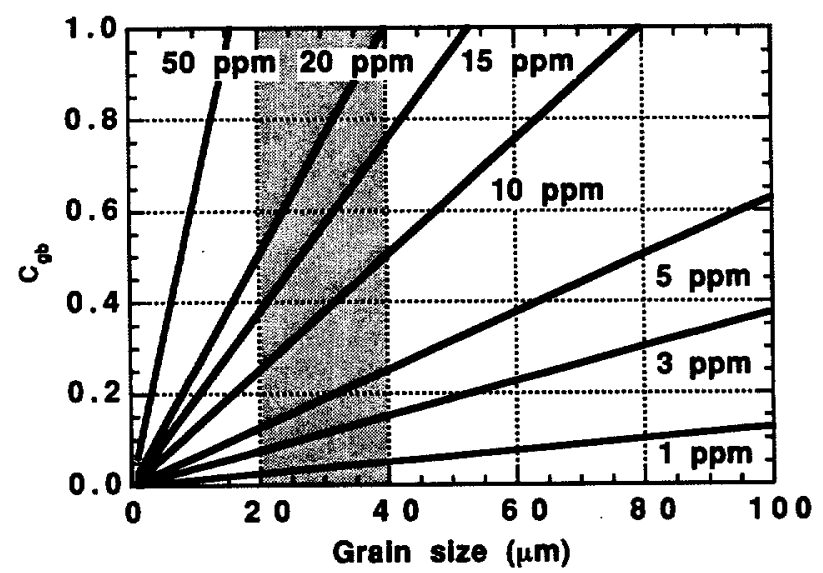

Figure 2. Calculated concentration of impurities at the grain boundary as a function of grain size. Different levels of bulk impurity content are plotted. The shaded area represents the region of interest of grain size and impurity content for the liners in this investigation.

Triple Line Impurity Concentration: The calculation is performed by determining the number of impurity atoms per unit length times the total length of triple lines. As three grain boundaries intersect at a triple line, it is assumed that each contributes one atom per length of triple line. The number of atoms per length equals $3 / 2 r_{s}$, and the total number of triple line sites equals the total length of triple lines times the number of atoms per length. Therefore, 


$$
\begin{aligned}
& \text { Sites }_{\mathrm{tl}}=12 \lambda \cdot 3 / 2 \mathrm{r}_{\mathrm{s}}=\frac{18 \mathrm{~d}}{\sqrt{10} \mathrm{r}_{\mathrm{s}}} \text {, and } \\
& \mathrm{C}_{\mathrm{tl}}=\frac{\mathrm{N}_{\mathrm{i}}}{\text { Sites }_{\mathrm{tl}}}=\frac{8 \sqrt{2} \mathrm{r}_{\mathrm{s}} \mathrm{C}_{\mathrm{s}} \mathrm{d}^{2}}{45 \mathrm{a}_{\mathrm{o}}^{3}}
\end{aligned}
$$

The triple lines will saturate with impurities when $C_{t l}=1.0$ and $C_{s}=1 \times 10^{-6}$ for Eq. (12). The saturation grain size equals $1.22 \mu \mathrm{m}$ at $1 \mathrm{ppm}$ based on the calculations above, and $0.39 \mu \mathrm{m}$ at $10 \mathrm{ppm}$.

Quadruple Node Impurity Concentration: Four triple lines come together at a quadruple node. Each triple line contributes 3 impurity atoms. Therefore, at each quadruple node, there are 12 impurity sites times 6 quadruple nodes per tetrakaidecahedron, for a total of 72 impurity sites.

$$
\mathrm{C}_{\mathrm{qn}}=\frac{\mathrm{N}_{\mathrm{i}}}{\text { Sites }_{\mathrm{qn}}}=\frac{2 \mathrm{C}_{\mathrm{s}} \mathrm{d}^{3}}{45 \sqrt{5} \mathrm{a}_{\mathrm{o}}^{3}}
$$

To calculate the grain size at which the concentration of impurities at the quadruple nodes saturates at $1 \mathrm{ppm}$ bulk impurity content, set $\mathrm{C}_{\mathrm{qn}}=1.0$ and $\mathrm{C}_{\mathrm{s}}=1 \mathrm{ppm}\left(1 \times 10^{-6}\right)$. Thus, saturation at the quadruple nodes occurs for grain sizes greater than $133.64 \mathrm{~nm}$ at $1 \mathrm{ppm}$ bulk impurity concentration.

\section{APPLICATION TO SHAPED CHARGE EXPERIMENTAL DATA}

This section evaluates the experimental shaped charge data in terms of the analysis described above. Using the two experimentally determined parameters, grain size and bulk impurity content, the analysis examines the correlation between the grain boundary impurity concentration and the jet ductility as measured by breakup time. Table 2 lists the breakup time as a function of impurity content and grain size, where the grain size is the average value measured by Chan [2].

Table 2. Bulk sulfur content, grain size, breakup time, and grain boundary impurity content for the liners shot in this investigation.

\begin{tabular}{|c|c|c|c|}
\hline $\begin{array}{c}\text { Sulfur } \\
\text { concentration } \\
(\mathrm{ppm})\end{array}$ & $\begin{array}{c}\text { Grain size, } \mathrm{d} \\
(\mu \mathrm{m})\end{array}$ & $\begin{array}{c}\text { Breakup time } \\
(\mu \mathrm{sec})\end{array}$ & $\mathrm{C}_{\mathrm{gb}}$ \\
\hline 3 & 40 & 186 & 0.151 \\
\hline 4 & 40 & 185 & 0.201 \\
\hline 7 & 40 & 147 & 0.353 \\
\hline 8 & 40 & 148 & 0.403 \\
\hline 9 & 40 & 152 & 0.453 \\
\hline 10 & 20 & 193 & 0.252 \\
\hline 15 & 20 & 160 & 0.378 \\
\hline 15 & 20 & 161 & 0.378 \\
\hline
\end{tabular}

The breakup time of sulfur-doped ofe copper is plotted as a function of grain size and bulk sulfur content in Figure 3(a and b). There does not appear to exist a straightforward correlation between the breakup time and the sulfur content or grain size. Fig. 3(a) reveals a somewhat counter-intuitive behavior, i.e., within the band of higher breakup times, the higher sulfur containing liner (10 ppm) had better jet ductility than the lower sulfur content liners ( 3 and 4 ppm). Similarly, in the collection of data with lower breakup times, the higher sulfur containing liners (15 ppm) had better breakup times than the lower sulfur liners ( 7 - $9 \mathrm{ppm})$. 

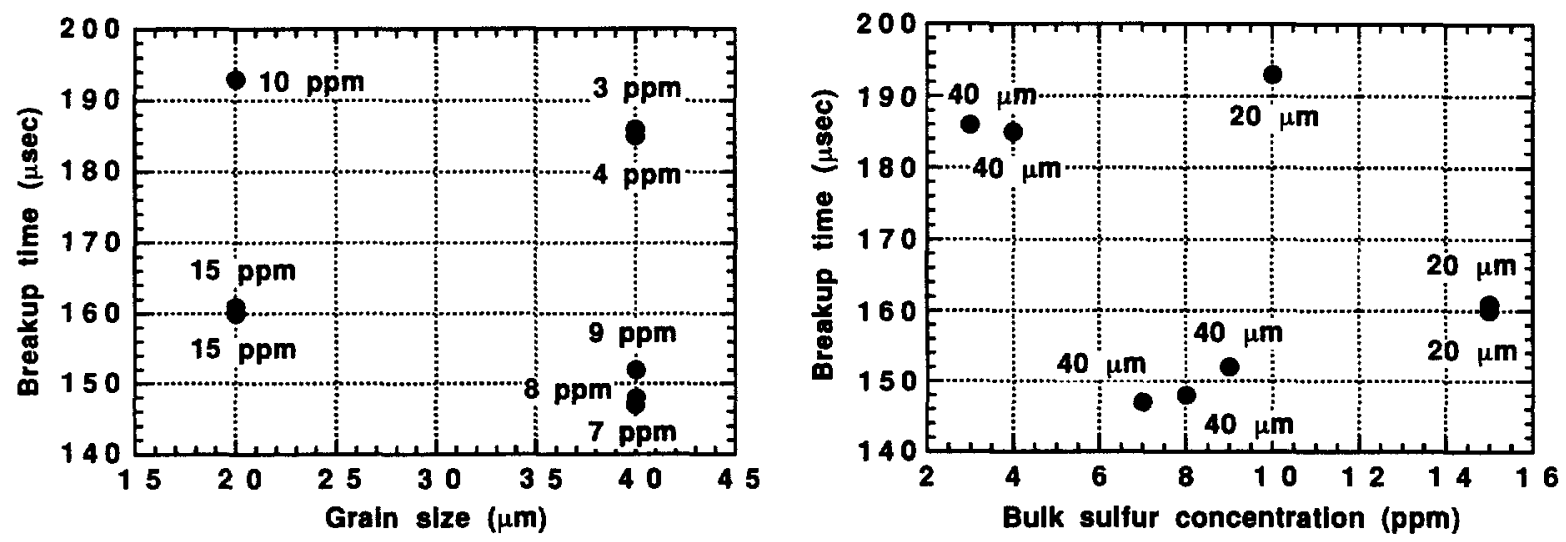

Figure 3. (a) Plot of breakup time as a function of grain size. (b) Breakup time as a function of bulk sulfur content. These two plots reveal no systematic relationship among breakup time, sulfur content, and grain size.

The relationship among the breakup time, grain size, and bulk impurity content of the liners in Table 2 is now analyzed in terms of Eq. (10). Substitution of the experimentally determined grain size and impurity concentration allows the breakup time to be plotted in Figure 4 as a function of the sulfur content at the grain boundaries. The figure indicates that the concentration spans the range from approximately $15 \%$ coverage to nearly $45 \%$ coverage of the boundaries in the monolayer geometry described previously.

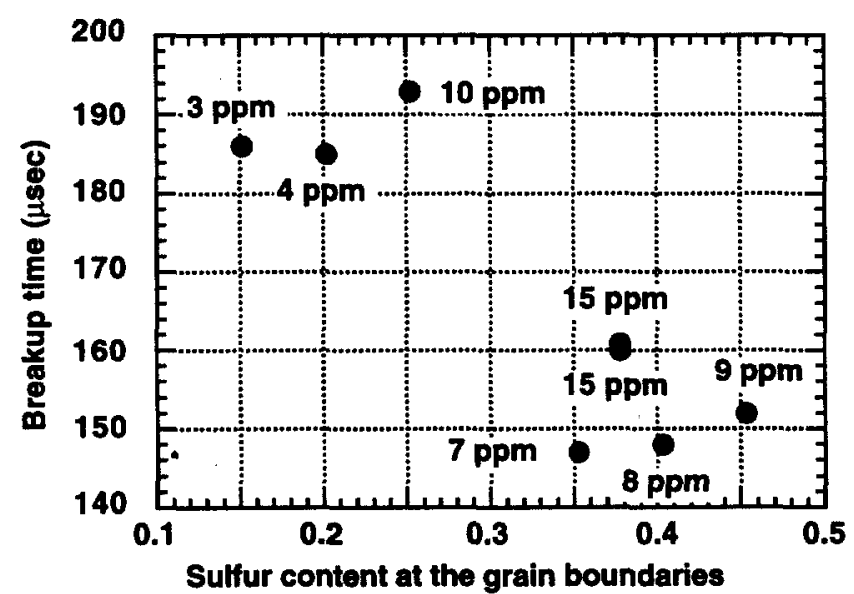

Figure 4. Plot of breakup time as a function of sulfur content at the grain boundaries for $40 \mu \mathrm{m}$ and $20 \mu \mathrm{m}$ grain sizes, (1.0 represents a full monolayer of coverage). The breakup time decreases with increasing grain boundary impurity concentration.

X-ray radiographs of four shaped charge liner shots are shown in Figure 5. Fig. 5(a,b) are 40 $\mu \mathrm{m}$ grain size liners with 3 and $7 \mathrm{ppm}$ sulfur levels. Figure 5(c,d) represent $20 \mu \mathrm{m}$ liners with $10 \mathrm{ppm}$ and $15 \mathrm{ppm}$ sulfur, respectively.

\section{DISCUSSION}

Viewed outside the context of a combined influence of grain size and bulk impurity content, the interpretation of the radiographs in Figure 5 would be misleading. Impurity content, examined independently, cannot explain the more ductile behavior of the 10 and $15 \mathrm{ppm}$ sulfur jets compared with the $7 \mathrm{ppm} S$ radiograph. An independent examination of the grain size alone would also present misleading information. Both the 3 and $7 \mathrm{ppm} \mathrm{S}$ liners have the same grain size, but dramatically different breakup times and jet characteristics. This variability in jet behavior is revealed clearly in Figure 3, which suggests no straightforward interpretation of the correlation between breakup time, grain size, and bulk impurity content. However, when the 
breakup times and radiographs are examined in light of Eq. (10), a reasonable hypothesis can be presented. The application of Eq. (10) to the grain size and bulk impurity content of the liners given in Table 2 indicates that the grain boundaries have impurity coverages between 0.15 and 0.45 of a monolayer, a significant enough level to expect an influence on jet ductility. The plot of breakup time as a function of grain boundary impurity concentration in Figure 4 as well as the radiographs in Figure 5 demonstrate that there exists a combined influence of grain size and bulk impurity content on the jet ductility. Thus, the analysis strongly suggests that the grain boundary impurity content is a reliable predictor of the shaped charge jet ductility.
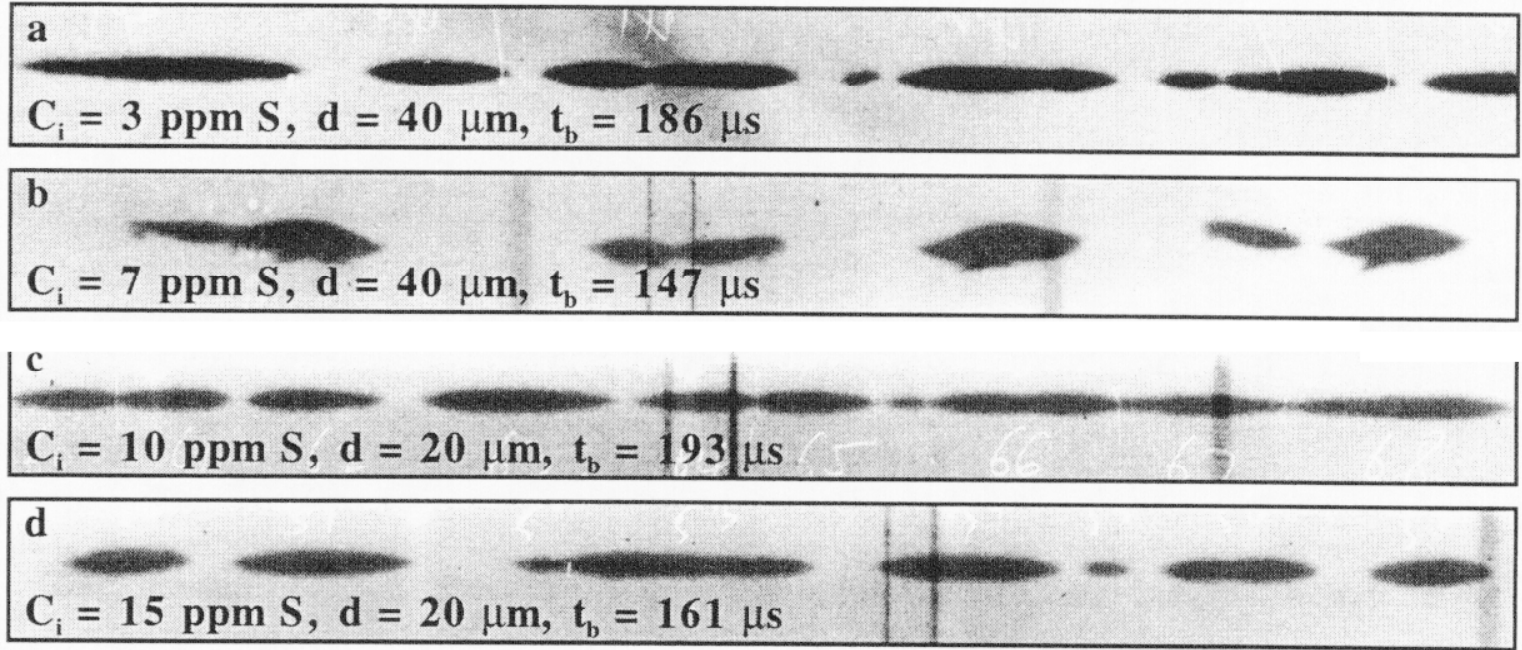

Figure 5. X-ray radiographs of sulfur-doped ofe $\mathrm{Cu}$ liners. (a) $3 \mathrm{ppm} \mathrm{S,} 40 \mu \mathrm{m}$ grain size, (b) $7 \mathrm{ppm}, 40 \mu \mathrm{m}$ grain size, (c) $10 \mathrm{ppm}, 20 \mu \mathrm{m}$ grain size, and (d) $15 \mathrm{ppm}, 20 \mu \mathrm{m}$ grain size.

Despite predicting decreasing ductility with increasing grain boundary impurity content, the analysis above implies that the jet ductility (breakup time) would continue to decrease with the $\mathrm{C}_{\mathrm{gb}}$. This behavior over the entire spectrum of grain boundary impurity concentrations does not appear physically likely in light of the transition from ductile, to semi-brittle or brittle jet behavior, to liner particulation. A more likely scenario may be as follows:

(i) At low $\mathrm{C}_{\mathrm{gb}}$, the jet behavior is ductile with high aspect ratio particles.

(ii) At medium $\mathrm{C}_{\mathrm{gb}}$, the behavior transitions to relatively high aspect ratio particles with some evidence of brittle fracture. Void nucleation likely occurs at the quadruple nodes, triple lines, and grain boundaries.

(iii) As $\mathrm{C}_{g b}$ continues to increase, the majority of the particles appear to break up in a semibrittle, or brittle fashion. Void nucleation occurs readily at the grain boundaries.

(iv) As $\mathrm{C}_{g b}$ approaches one, there likely exists a critical $\mathrm{C}_{\mathrm{gb}}$ at which the jet behavior transitions from brittle jet breakup to jet particulation. At this stage, it is possible that second phase particles occur at the grain boundaries.

This scenario assumes a homogeneous distribution of impurity atoms at every grain boundary, and that every grain boundary behaves similarly under high strain rate, rapid temperature rise deformation. This is not likely an accurate assumption in that impurity atoms are more prone to segregate to random, and high $\Sigma$ boundaries than to low angle and low $\Sigma$ boundaries. The coincident site lattice (CSL) model has been used to categorize the misorientation of grain boundaries [12]. In this model, the $\Sigma$ value corresponds to the inverse of the number of lattice sites in the coincident site lattice. Small $\Sigma$ s correspond to high coincidence, low mismatch boundaries such as twins, and other low energy boundaries. The analysis above should then be modified to assume that some high $\Sigma$ boundaries may approach saturation (depending on the overall value of $C_{g b}$ ), whereas low $\Sigma$ boundaries remain less concentrated. To validate this argument, a detailed understanding of the location of the impurity atoms at different types of boundaries is required, in addition to the grain boundary character distribution which describes the relative fractions of high and low $\Sigma$ boundaries. 


\section{SUMMARY}

1. Tetrakaidecahedron grain shapes were used as an approximation of copper microstructures. The use of tetrakaidecahedra allows for straightforward calculations of grain volume, surface area, triple line length, and number of quadruple nodes.

2. By assuming that all the impurity atoms segregate to, and remain at, the quadruple nodes, triple lines, and grain boundaries, the intercrystalline impurity concentrations at these locations can be calculated. The concentration of impurity atoms at quadruple nodes is proportional to (grain size) ${ }^{3}$, triple lines proportional to (grain size) ${ }^{2}$, and the concentration of impurity atoms at grain boundaries is proportional to (grain size) ${ }^{1}$.

3. Quadruple nodes and triple lines are saturated at grain sizes of interest in conventionally processed shaped charge liner materials. Grain boundaries are only partially filled with impurity atoms.

4. The breakup time of copper shaped charge liners doped with sulfur is observed to decrease with the grain boundary impurity content.

The results of this study suggest that the transition from semi-brittle or brittle breakup to particulation may be due to some critical concentration of impurities at the grain boundaries, possibly near saturation. The analysis also suggests that the distribution of impurities at special versus random boundaries in conjunction with the grain boundary character distribution may play an important role in the breakup behavior.

\section{ACKNOWLEDGMENTS}

This work performed under the auspices of the U.S. Department of Energy and Lawrence Livermore National Laboratory under contract No. W-7405-Eng-48.

\section{REFERENCES}

[1] M.L. Duffy and S.T. Golaski, "Effect of Liner Grain Size on Shaped Charge Jet Performance and Characteristics," U.S. Army Ballistic Research Laboratory Technical Report No. BRL-TR-2800, (1987).

[2] D.K. Chan, D.H. Lassila, W.E. King, and E.L. Baker; "MRS Proceedings on Fracture Instability Dynamics, Scaling and Ductile/Brittle Behavior," R.L. Blumberg Selinger, J.J. Mecholsky, A.E. Carlsson, and E.R. Fuller, Eds., Mater. Res. Soc., 195-200 (1996).

[3] D.H. Lassila, E.L. Baker, D.K. Chan, W.E. King and A.J. Schwartz, "Effect of Sulfur on the Ductility of Copper Shaped-Charge Jets," Proc. 16th Int'l Ballistics Symposium, San Francisco, CA, $31-38$ (1996).

[4] A. Lichtenberger, "Some Criteria for the Choice of Shaped Charge Copper Liners," Proc. 11th Int'l Ballistics Symposium, Brussels, Belgium, 5-11 (1989).

[5] A. Lichtenberger, "The Influence of Grain size and Quality of Nickel-Based Liners on the Ductility of Shaped Charge Jets," 13th Ballistics, Stockholm, Sweden, 337-384 (1992).

[6] T.G. Nieh and W.D. Nix, "Embrittlement of Copper Due to Segregation of Oxygen to Grain Boundaries," Met. Trans. A, 12A, 893-901 (1981).

[7] D.H. Lassila, "Correlations Between Shaped Charge Jet Breakup and Grain Boundary Impurity Concentrations," 13th Int'l Ballistics, Stockholm, Sweden, 549-556 (1992).

[8] A. Fraczkiewicz and M. Biscondi, "Intergranular Segregation of Bismuth in Copper Bicrystals," Journal de Physique, C4, S4, 497-503 (1985).

[9] G. Palumbo, S.J. Thorpe, and K.T. Aust, "On the Contribution of Triple Junctions to the Structure and Properties of Nanocrystalline Materials," Scripta Mat., 24, 1347 (1990).

[10] N. Wang, G. Palumbo, Z. Wang, U. Erb, K.T. Aust, "On the Persistence of Four-Fold Triple Line Nodes in Nanostructured Materials," Scripta Met et Mat, 28, 253-256 (1993).

[11] Interstitial impurities were measured by Luvak, Inc., metallic impurities were measured by Northern Analytical Laboratory Inc. using glow discharge mass spectrometry.

[12] H. Grimmer, W. Bollmann, and D.H. Warrington, "Coincidence-Site Lattices and Complete Pattern-Shift Lattices in Cubic Crystals," Acta Cryst. 30A, 197-207 (1974). 


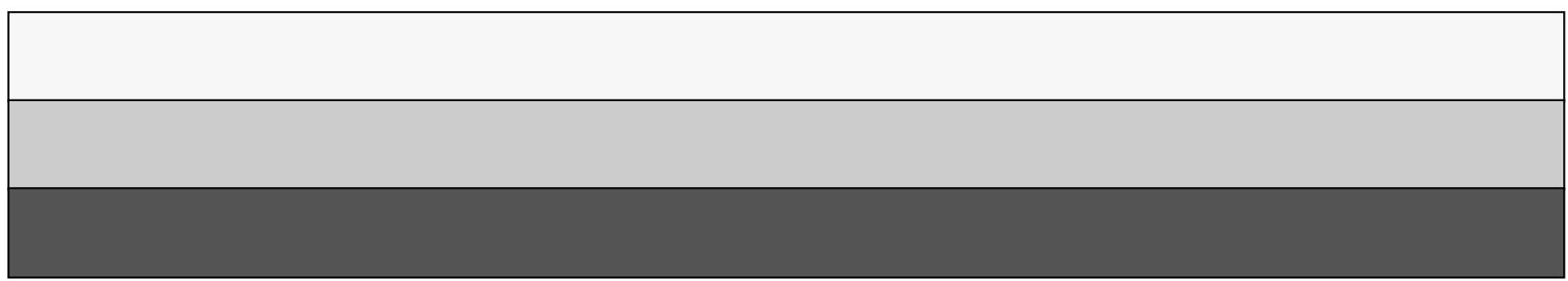

